\title{
ON THE DENJOY-PERRON-BOCHNER INTEGRAL
}

\author{
STANISEAW SIUDUT
}

\begin{abstract}
The notion of Denjoy integrals of abstract functions was first introduced by $A$. Alexiewicz [1]. His descriptive definitions are based upon a concept of the approximate derivative. In this paper we present another descriptive definition for the Denjoy-Perron integral of abstract functions - via the parametric derivative of Tolstov [8]. Some properties of this integral are examined.
\end{abstract}

We assume that the reader is familiar with [2]. Let $X$ be a Banach space. The definition of Tolstov ([8] p.387) and the important theorem of Armstrong ([2] p.36, Theorem 2) lead to the following definition.

Definition. Let $F:[a, b] \rightarrow X$, where $[a, b]$ is a finite interval. The function $f:[a, b] \rightarrow X$ is called the parametric derivative of $F$ if there exists a differentiable strictly increasing function $\varphi$ mapping $[\alpha, \beta]$ onto $[a, b]$ such that

$$
\frac{d}{d t} F(\varphi(t))=\varphi^{\prime}(t) \cdot f(\varphi(t)) \quad \text { for every } \quad t \in[\alpha, \beta] .
$$

The function will be called a dpr for $F$ (dpr stands for differentiable parametric representation). We shall write $D(F)=f$.

The properties of the parametric derivative are the same as in [2], namely we have

a. If $F^{\prime}=f$ then $\varphi(t)=t$ is a dpr for $F$.

b. If $F$ has a parametric derivative, then it is continuous.

c. If $D(F)=f$, and $F$ has dpr $\varphi$, and $\varphi^{\prime}(t) \neq 0$, then $F$ has an ordinary derivative at the point $x=\varphi(t), F^{\prime}(t)=f(x)$.

d. If $D(F)=f$ on $[a, b]$, then a.e. on $[a, b] F$ has an ordinary derivative $F^{\prime}=f$ (cit. [2] pp.31, 32).

The proofs of the above properties run as in [2]. The properties 1, 2, 3 ([2] p.31) remain also true, i.e. we have

Received April 29, 1993; revised September 23, 1993.

1991 Mathematics Subject Classification. 46G10, 26A39

Key words and phrases. Denjoy-Bochner integral, differentiable parametric representation. 
1. $D(k F)=k D(F), k$ is a constant,

2. $D(F+G)=D(F)+D(G)$

3. $D(F)=0$ implies $F$ is a constant.

To prove 2 define $\Theta=\left(\left(\varphi^{-1}+\psi^{-1} / 2\right)^{-1}\right.$, where $\varphi, \psi$ are dpr for $F$, $G$, respectively. For every $h$ belonging to the set $\left\{\varphi^{-1}, \psi^{-1}, \Theta^{-1}\right\} h$ is a strictly increasing bijection $[a, b]$ onto $[\alpha, \beta]$ and $0<h^{\prime} \leq \infty$, so $0 \leq\left(h^{-1}\right)^{\prime}<\infty$ and $h^{-1}$ is a strictly increasing bijection $[\alpha, \beta]$ onto $[a, b]$. Thus $\Theta$ satisfies the assumptions of our definition. Obviously we have $0<2\left(\Theta^{-1}\right)^{\prime}=\left(\varphi^{-1}\right)^{\prime}+\left(\psi^{-1}\right)^{\prime} \leq \infty$, hence $\left(\Theta^{-1}\right)^{\prime}(x)=\infty$ if and only if $\left(\varphi^{-1}\right)^{\prime}(x)=\infty$ or/and $\left(\psi^{-1}\right)^{\prime}(x)=\infty$. Therefore, if $x=\Theta(t)=\varphi(s)=\psi(r)$ then we obtain

$$
\Theta^{\prime}(t)=0 \text { if and only if } \varphi^{\prime}(s)=0 \quad \text { or } / \text { and } \quad \psi^{\prime}(r)=0
$$

The above, the Definition and property $c$ imply that $\Theta$ is a dpr for both $F$ and $G$, because $(F \circ \Theta)^{\prime}(t)$ and $(G \circ \Theta)^{\prime}(t)$ exist. To prove the last statement we consider for example the case $\Theta^{\prime}(t)=0, \varphi^{\prime}(s)=0, \psi^{\prime}(r) \neq 0$. Since $x=\Theta(t)=\varphi(s)=\psi(r)$ and $\Theta(t+h)=$ $\Theta(t)+o(h), \varphi\left(s+h_{1}\right)=\varphi(s)+o_{1}\left(h_{1}\right), \psi\left(r+h_{2}\right)=\psi(r)+\psi^{\prime}(r) h_{2}+o_{2}\left(h_{2}\right)$, we obtain for $\Theta(t+h)=\varphi\left(s+h_{1}\right)=\psi\left(r+h_{2}\right)$ the following equalities $o(h)=o_{1}\left(h_{1}\right)=o_{2}\left(h_{2}\right)+\psi^{\prime}(r) h_{2}$. According to the definition of $\Theta$ we get $t=(s+r) / 2, t+h=\left(s+h_{1}+r+h_{2}\right) / 2$ and the signs of $h, h_{1}, h_{2}$ are the same. Therefore $2 h=h_{1}+h_{2}$ and $h_{1} / h$ is bounded as $h \rightarrow 0$ (moreover, $h \rightarrow 0$ if and only if $h_{1} \rightarrow 0$ ). Consequently,

$$
\begin{aligned}
(F \circ \Theta)^{\prime}(t) & =\lim _{h \rightarrow 0}\left(F(x+o(h)-F(x)) / h=\lim _{h \rightarrow 0} \frac{h_{1}}{h} \cdot \frac{F\left(x+o_{1}\left(h_{1}\right)\right)-F(x)}{h_{1}}\right. \\
& =\lim _{h \rightarrow 0} \frac{h_{1}}{h} \cdot \frac{F\left(\varphi\left(s+h_{1}\right)\right)-F(\varphi(s))}{h_{1}}=0,
\end{aligned}
$$

because of $(F \circ \varphi)^{\prime}(s)=0$. This proves the existence of $(F \circ \Theta)^{\prime}(t)$. The existence of $(G \circ \Theta)^{\prime}(t)$ is a consequence of the property $c$.

Finally, $\Theta$ is a dpr for both $F$ and $G$. Thus $((F+G) \circ \Theta)^{\prime}=(F \circ \Theta+G \circ \Theta)^{\prime}=$ $(F \circ \Theta)^{\prime}+(G \circ \Theta)^{\prime}$, which yields property 2 (cf. [2], p. 32). Properties 1 and 3 are easy to prove (cf. [2], pp. 31, 32).

Let $X_{i}(i=1,2,3)$ be a Banach space, $F_{i}:[a, b] \rightarrow X_{i}(i=1,2), B: X_{1} \times X_{2} \rightarrow X_{3}$ is an arbitrary bilinear and continuous map. Then we have also

e. $D\left(B\left(F_{1}, F_{2}\right)\right)=B\left(F_{1}, D\left(F_{2}\right)\right)+B\left(D\left(F_{1}\right), F_{2}\right)$.

The proof is similar to that of [2] p.32 if we use a formula on the derivative of bilinear product from [7], Chapter III, 4.

Let $f$ be a parametric derivative of $F$ on $[a, b]$. We define the Denjoy-Perron-Bochner integral (short: DPB-integral) of $f$ by

$$
\int_{a}^{b} f=F(b)-F(a)
$$


(see [2] p.32 (3) for real-valued functions). The function $f$ above is said to be DenjoyPerron-Bochner integrable (short: DPB-integrable). This integral is well-defined (see the proof of Theorem 1, [2] p.33). Moreover, theorem 2, 3, 4, 5 from [2] pp. 33, 34 remain also true with the similar proofs (obviously the proof of 4 must be suitable changed). Therefore the following properties hold for DPB integral:

$\alpha$. if $f, g$ are DPB-integrable in $[a, b], k$ is a constant, then $k f, f+g$ are also DPBintegrable on $[a, b]$ and

$$
\int_{a}^{b} k f=k \int_{a}^{b} f, \quad \int_{a}^{b}(f+g)=\int_{a}^{b} f+\int_{a}^{b} g
$$

$\beta$. if $f$ is DPB-integrable in $[a, c]$ and on $[c, b]$, then $f$ is DPB-integrable on $[a, b]$ and

$$
\int_{a}^{b} f=\int_{a}^{c} f+\int_{c}^{b} f
$$

$\gamma$. if $F_{1}, F_{2}$ are the same as in the property $d, D\left(F_{1}\right), D\left(F_{2}\right)$ exist and one of summands on the right side of $d$ is DPB-integrable, so is the other, and

$$
\left.\int_{a}^{b} B\left(F_{1}, D\left(F_{2}\right)\right)=B\left(F_{1}(b), F_{2}(b)\right)-B\left(F_{1}(a), F_{2}(a)\right)-\int_{a}^{b} B\left(D\left(F_{1}\right), F_{2}\right)\right),
$$

$\delta$. if $f$ is the derivative of $F$ on $[a, b]$, then $f$ is DPB-integrable and

$$
\int_{a}^{b} f=F(b)-F(a)
$$

In the sequel the Bochner integral of $f$ on $[a, b]$ will be denoted by $(B) \int_{a}^{b} f$. Using Th. 8 of Zahorski ([10] p.35) and arguing as in [2] p.35 we obtain

$\varepsilon$. if $f=0$ a.e. on $[a, b]$, then $f$ is DPB-integrable on $[a, b]$ and $\int_{a}^{b} f=0$. Now, we shall prove the following

Theorem A. A DPB-integrable function $f$ is strongly measurable.

Proof. Let $f=D(F)$ be defined on $[a, b]$, and $Z=\left\{x \in(a, b): F^{\prime}(x)\right.$ exists and $\left.F^{\prime}(x)=f(x)\right\}$. By the property $d$, the complement of the set $Z$ to the set $[a, b]$ has Lebesgue measure zero. Define $h_{n}=(b-a) / n, I(n, k)=\left[a+(k-1) h_{n}, a+\right.$ $\left.k h_{n}\right], \Delta I(n, k)=h_{n}, \Delta F(n, k)=F\left(a+k h_{n}\right)-F\left(a+(k-1) h_{n}\right), y_{n}(x)=\sum_{k=1}^{n} \chi_{n, k}(x)$ $\triangle F(n, k) / \triangle I(n, k)$, where $\chi_{n, k}$ is the characteristic function of the interval $I(n, k)$.

Fix $x \in Z$. For every $n$ there exists an interval $I(n, k(t))$ containing $t$, where $t$ is a member of $(a, b)$. Moreover, the diameter of this interval tends to zero as $n$ tends to infinity. Thus

$$
\lim _{n \rightarrow \infty} \frac{\triangle F(n, k(x))}{\Delta I(n, k(x))}=F^{\prime}(x) \quad(\text { see [5] p.157). }
$$


Consequently, the sequence of simple functions $y_{n}$ tends to $F^{\prime}(x)=f(x)$ for almost all $x \in[a, b]$ and therefore $f(x)$ is strongly measurable on $[a, b]([9]$ p. 130).

Theorem B. If $f:[a, b] \rightarrow X$, the dimension of $X$ is finite and $f$ is Bochner integrable on $[a, b]$, then $f$ is DPB-integrable there and the two integrals are equal.

Proof. Let $e_{1}, \ldots, e_{n}$ be a basis of $X$ and $f=\sum_{i=1}^{n} f_{i} e_{i}$. From this and T'h. 3 [2] it follows that

$$
\text { (B) } \int_{a}^{b} f=\sum_{i=1}^{n}\left((L) \int_{a}^{b} f_{i}\right) \cdot e_{i}=\sum_{i=1}^{n}\left(\int_{a}^{b} f_{i}\right) e_{i}=\sum_{i=1}^{n} \int_{a}^{b}\left(f_{i} e_{i}\right)=\int_{a}^{b} f
$$

by the property 2 and $e$.

Theorem C. Let $T$ be a bounded linear operator on a Banach space $X$ into a Banach space $Y$. If $f$ is an $X$-valued and DPB-integrable on $[a, b]$ function, then $T f$ is a $Y$-valued DPB-integrable function, and

$$
T\left(\int_{a}^{b} f\right)=\int_{a}^{b} T \circ f
$$

Proof. Let $F$ be a parametric primitive of $f$ with $\operatorname{dpr} \varphi$. Now $T$ is continuous and therefore it is commutative with the derivative. We have $((T \circ F) \circ \varphi)^{\prime}(t)=(T \circ(F \circ$ $\varphi))^{\prime}(t)=T\left((F \circ \varphi)^{\prime}(t)\right)=T\left(\varphi^{\prime}(t) f(\varphi(t))\right)=\varphi^{\prime}(t) \cdot[T \circ f](\varphi(t))$, whence $\varphi$ is a dpr for $T \circ F$ and $D(T \circ F)=T \circ f$. Thus

$$
\int_{a}^{b} T \circ f=(T \circ F)(b)-(T \circ F)(a)=T(F(b)-F(a))=T\left(\int_{a}^{b} f\right)
$$

and the proof is finished.

Theorem D. If $f$ is Bochner integrable on $[a, b]$ and $f$ is DPB-integrable on $[a, b]$, then the two integrals are equal there.

Proof. Denote the first integral by $u$, the second one by $v$ both on $[a, b]$. Let $T$ be a bounded linear functional defined on $X$.

Define

$$
p=(B) \int_{a}^{b} T \circ f, \quad q=\int_{a}^{b} T \circ f .
$$

It follows from Th. C, Corollary 2 of [9] p. 134 and Th. 3 of [2] that

$$
T(u-v)=T u-T v=p-q=0 .
$$

Therefore $u-v$ must be 0 (since $T$ is an arbitrary element of the dual space of $X$ ). The proof is finished. 
From the descriptive definitions of the Bochner and the Denjoy-Bochner integrals ([1], pp. 101, 102) it follows immediately that if $f$ is Bochner integrable on $[a, b]$, then $f$ is DB-integrable there and the two integrals are equal. But then the DB-integral is a generalization of the general Denjoy integral, whereas our DPB-integral is a generalization of the restricted Denjoy integral. These considerations and Theorems B, D suggest the following theorem.

Theorem E. If $f$ is Bochner integrable on $[a, b]$, then $f$ is DPB-integrable there and the two integrals are equal.

Proof. Let \|\| be the norm in the space $X, A=(B) \int_{a}^{b}\|f(x)\| d x$. If $A=0$, then $\|f(x)\|=0$ for almost all $x$ in $I=[a, b]$, so $f=0$ a.e. on $I$. Thus the two integrals are equal by the property $\varepsilon$ (see also [9] p. 133).

Suppose $A>0$. Since the function $c(t)=t+(B) \int_{a}^{t}\|f(x)\| d x$ is increasing and continuous on $I$, then for every $s$ from $J=[a, b+A]$ there exists $t \in I$, such that $s=c(t)$. Denoting this $t$ by $d(s)$, we have the increasing bijection $d: J \rightarrow I$. Let us consider the function $F(t)=(B) \int_{a}^{t} f(x) d x$. Taking $s_{1} \leq s_{2}$ from $J$, we obtain for $t_{i}=d\left(s_{i}\right),(i=1,2)$

$$
\left|d\left(s_{2}\right)-d\left(s_{1}\right)\right|=t_{2}-t_{1} \leq t_{2}-t_{1}+(B) \int_{t_{1}}^{t_{2}}\|f(x)\| d x=\left|s_{2}-s_{1}\right|,
$$

and similarly

$$
\left\|F\left(d\left(s_{2}\right)\right)-F\left(d\left(s_{1}\right)\right)\right\|=\left\|(B) \int_{t_{1}}^{t_{2}} f(x) d x\right\| \leq t_{2}-t_{1}+(B) \int_{t_{1}}^{t_{2}}\|f(x)\| d x=\left|s_{2}-s_{1}\right| .
$$

From this it follows that the funcitons $d, F \circ d$ satisfy the Lipschitz condition on $J$. Moreover, $d$ (as well as $c$ ) is absolutely continuous.

The set $E=\left\{x \in I: F^{\prime}(x)=f(x)\right\}$ has measure $b-a$, thus the set $Z=I \backslash E$ has measure zero (Th. 2, [9], p. 134). Therefore the set $d^{-1}(Z)=c(Z)$ has measure zero (Th. 1, [5] p. 172) and $(F \circ d)^{\prime}(s)$ exists for almost all $s \in J$. Indeed, $d^{\prime}(s)$ exists for almost all $s \in J$ and therefore $(F \circ d)^{\prime}(s)=F^{\prime}(d(s)) \cdot d^{\prime}(s)$ for almost all $s \in J \backslash d^{-1}(Z)$, thus for almost all $s \in J$ (cf. [5], Corollary, p. 173). Repeating the arguments of Bruckner ([4], p. 555, lines 17,21 ) we can find a $\operatorname{dpr} \varphi$ for $F \circ d$ and a $\operatorname{dpr} \psi$ for $d$ (both $F \circ d$ and $d$ satisfy the Lipschitz condition). Let $\Theta: K \rightarrow J$ be a common dpr for $F \circ d, d$ (compare with the proof of property 2). Thus

$$
(F \circ d \circ \Theta)^{\prime}(r) \text { exists for all } r \in K
$$

Denote by $C, G$ the sets

$$
\left\{x \in I \backslash E: x=d \circ \Theta(r),(d \circ \Theta)^{\prime}(r)=0 \quad \text { for some } \quad r \in K\right\},
$$




$$
\left\{x \in I \backslash E: x=d \circ \Theta(r),(d \circ \Theta)^{\prime}(r) \neq 0 \quad \text { for some } \quad r \in K\right\},
$$

respectively. The sets $E, C, G$ are disjoint and $E \cup C \cup G=I$, since $(d \circ \Theta)^{\prime}(r)$ exists for all $r \in K$. Define the function $g(x)$ to be $f(x), 0,\left((d \circ \Theta)^{\prime}(r)\right)^{-1}$. $(F \circ d \circ \Theta)^{\prime}(r)$ for $x=d \circ \Theta(r)$ belonging to $E, C, G$, respectively. From this definition and (1) we obtain

$$
(F \circ(d \circ \Theta))^{\prime}(r)=g(d \circ \Theta(r)) \cdot(d \circ \Theta)^{\prime}(r) \text { for all } r \in K,
$$

and therefore $d \circ \Theta$ is a dpr for $F, D(F)=g$. Since $g-f=0$ a.e. on $I$, then $g-f$ is DPB-integrable and its DPB-integral is equal to zero (property $\varepsilon$ ). Finally,

$$
\begin{gathered}
F(b)-F(a)=\int_{a}^{b} g=\int_{a}^{b}(f-g+g)=\int_{a}^{b} f \quad \text { (property } \alpha \text { ), so } \\
(B) \int_{a}^{b} f(x) d x=F(b)-F(a)=\int_{a}^{b} f, \quad \text { (q.e.d.) }
\end{gathered}
$$

The theorem on integration by substitution ([2], p.38) can be reformulated as follows.

T'heorem $\mathbb{F}$. Let $g:[c, d] \rightarrow \mathbb{R}$ be Lebesgue integrable and positive on $[c, d]$, $G(t)=a+\int_{0}^{t} g$ for $c \leq t \leq d$, and $G(d)=b$. If $f:[a, b] \rightarrow X$ is DPB-integrable on $[a, b]$, then $g \cdot(f \circ G)$ is DPB-integrable on $[c, d]$ and $\int_{a}^{b} f=\int_{c}^{d} g \cdot(f \circ G)$.

The proof is similar to that of [2] p. 38, 39. Indeed, let us observe that our function $g$ is DPB-integrable (by Tolstov's theorem, [8]). In the diagram on p. 34, [2], we must take $X$ instead of $R$.

Example. Let $F(x)=x^{2} \sin \left(1 / x^{2}\right)$ for $x \neq 0, F(0)=0$ and $w \in X, w \neq 0$. The function $f=D(F) \cdot w$ is DPB-intergrable on $[0,1]$, but $f$ is not $B$-integrable on this interval. This remark is an obvious consequence of a Saks' example, see also [2] p. 35.

\section{References}

[1] A. Alexiewicz, "On Denjoy integrals of abstract functions," C. R. Soc. Sci. et Letters Varsovie, Classe III (1950), 97-129.

[2] G. M. Armstrong, "Properties of a general integral equivalent to the Denjoy-Perron integral," Tamkang J. Math. Vol. 17, No. 4(1986), 29-39.

[3] G. M. Armstrong, "A classical approach to the Denjoy integral by parametric derivatives," $J$. London Math. Soc. (2), 3(1971), 346-349.

[4] A. M. Bruckner, "Creating differentiability and destroying derivatives," Amer. Math. Monthly, Vol. 85 , No. $7(1978), 554-562$.

[5] S. Lojasiewicz, An introduction to the theory of real functions, (Polish), Warszawa 1973.

[6] S. Saks, Theory of the integral, Monografie Matematyczne, Warszawa-Lwów 1937.

[7] L. Schwartz, Cours d'analyse, Hermann, Paris 1981.

[8] G. P. Tolstov, "Parametric differentiation and the restricted Denjoy integral," (Russian), Mat. Sb., 53(1961), 387-392.

[9] K. Yosida, Functional Analysis, Third ed., Springer-Verlag, New York 1971.

[10] Z. Zachorski, "Sur la Première Dèrivèe," Trans. Amer. Math. Soc., 69(1950), 1-54. 